\title{
A hypothesis for the mechanism of urine incontinence in patients after radical prostatectomy due to urinary bladder hypertrophy
}

\author{
Kajetan Juszczak ${ }^{1, A-F}$, Adam Ostrowski2, ${ }^{2, B, D}$, Michał Bryczkowski ${ }^{2, A, B, D}$, \\ Przemysław Adamczyk ${ }^{3, A, B, D}$, Tomasz Drewa ${ }^{2,3, A, D-F}$ \\ ${ }^{1}$ Department of Urology, Ludwik Rydygier Memorial Specialized Hospital, Kraków, Poland \\ ${ }^{2}$ Department of General and Oncologic Urology, Nicolaus Copernicus University, Bydgoszcz, Poland \\ ${ }^{3}$ Department of General and Oncological Urology, Nicolaus Copernicus Hospital, Toruń, Poland \\ A - research concept and design; B - collection and/or assembly of data; $C$ - data analysis and interpretation; \\ $D$ - writing the article; $E$ - critical revision of the article; $F$ - final approval of the article
}

Address for correspondence

Kajetan Juszczak

E-mail:kajus13@poczta.onet.pl

Funding sources

None declared

Conflict of interest

None declared

\section{Acknowledgements}

The authors would like to thank Miss Izabella Ostrowski, currently studying neuroscience at the University of Vermont, Burlington, USA for English linguistic support.

Received on March 12, 2017

Reviewed on August 16, 2017

Accepted on November 8, 2017

Published online on August 29, 2018

\begin{abstract}
Prostate cancer is one of the most common neoplasms in elderly males in Europe and is rapidly becoming a major health burden throughout the world. Radical prostatectomy is a first-line treatment in the case of organ-confined prostate cancer and in selected cases of locally advanced disease. Recent studies have suggested that the urinary bladder wall thickness (BWT) is an important predictor of an overactive bladder (OAB). This article focuses on a hypothesis for the mechanism of urine incontinence in patients after radical prostatectomy due to urinary bladder hypertrophy. To verify the possible influence of changes in the urinary bladder structure on urine incontinence development, we designed an experiment in which patients with prostate cancer were qualified for radical prostatectomy. Our hypothesis that urinary bladder hypertrophy influences urine incontinence development in patients after radical prostatectomy may provide an insight toward the prevention and control of urine incontinence after surgery by oral pharmacotherapy or intravesical onabotulinumtoxinA injections in pre-radical prostatectomy mode.
\end{abstract}

Key words: urinary incontinence, hypertrophy, urinary bladder, prostatectomy, structural changes

Cite as

Juszczak K, Ostrowski A, Bryczkowski M, Adamczyk P, Drewa T. A hypothesis for the mechanism of urine incontinence in patients after radical prostatectomy due to urinary bladder hypertrophy. Adv Clin Exp Med. 2019;28(3):391-395. doi:10.17219/acem/79935

DOI

10.17219/acem/79935

Copyright

Copyright by Author(s)

This is an article distributed under the terms of the

Creative Commons Attribution Non-Commercial License

(http://creativecommons.org/licenses/by-nc-nd/4.0/) 


\section{Introduction}

Prostate cancer is the most common neoplasm in elderly males in Europe and is a major health burden, especially in countries with a higher proportion of elderly men in the general population. Epidemiological data suggests that the highest incidence of this cancer can be found in Northern and Western Europe (approx. 200 cases per 100,000 men). Moreover, a continuous increase of prostate cancer morbidity can be observed in Eastern and Southern Europe. ${ }^{1}$ Radical prostatectomy is a first-line treatment in the case of organ-confined prostate cancer. Additionally, surgical treatment should be considered an option in all patients with locally advanced disease. The goal of radical prostatectomy in any approach is the eradication of the disease while preserving continence and, whenever possible, potency. ${ }^{2}$ However, some groups of patients require an adjuvant therapy (e.g., radiotherapy) after radical prostatectomy due to the onset of poor prognostic factors, as well as in cases of biochemical recurrence. Despite this, the 10-year survival of patients after radical prostatectomy is very high, reaching survival rates of $95-100 \%$. Radical prostatectomy is associated with postoperative complications (e.g., erectile dysfunction, urinary incontinence, etc.). Most of the patients who experience urinary incontinence after radical prostatectomy often have stress urinary incontinence. However, in some cases, the post-surgical urinary incontinence can also manifest due to urgency caused by the development of an overactive bladder (OAB), which is defined as urge urinary incontinence. Recent clinical observations suggest that men can also develop OAB after radical prostatectomy. Moreover, due to storage lower urinary tract symptoms (LUTS) (frequency, urgency, etc.), many patients with OAB usually reduce fluid intake and limit daily activity to avoid the discomfort of the symptoms. ${ }^{3}$ These facts significantly affect the quality of life of patients who receive radical prostatectomy treatment.

\section{The hypothesis}

We hypothesized that urinary bladder hypertrophy influences urine incontinence development by causing $\mathrm{OAB}$ in patients with prostate cancer after radical prostatectomy. The primary outcome of the study is defined as an increased incidence of OAB with or without urge urinary incontinence in patients after radical prostatectomy, which is characterized by increased urinary bladder weight and wall thickness. The secondary outcome is that urinary bladder outlet obstruction (BOO) increases the risk of $\mathrm{OAB}$ with or without urge urinary incontinence in patients after radical prostatectomy.

\section{Evaluation of the hypothesis}

\author{
Radical prostatectomy \\ and urinary incontinence
}

Radical prostatectomy remains the standard treatment of choice in the cases of organ-confined prostate cancer and in selected cases of locally advanced prostate cancer. ${ }^{4}$ The radical prostatectomy procedure consists of removing the prostate gland and seminal vesicles, ideally with negative surgical margins. Thus, radical prostatectomy is associated with an increased risk of developing urinary incontinence. Urinary incontinence after radical prostatectomy represents a troublesome issue in some patients. Many factors determine urinary incontinence after radical prostatectomy, including anatomical and biological factors. The pathophysiology of post-surgical urinary incontinence is multifactorial. Excluding anatomical determinants (e.g., changes in the urethral sphincter complex and/or structures related to the membranous urethra, damage to neural components, etc.), some biological factors have been defined. Several clinical features seem to be crucial predictors of post-prostatectomy urinary incontinence, including age, body mass index (BMI), comorbidity index, lower urinary tract symptoms, and prostate volume. Urinary incontinence in patients after radical prostatectomy is primarily due to urethral sphincter incompetence and weakness, known as stress urinary incontinence. ${ }^{5-7}$ Urge urinary incontinence, in some cases, can also be due to urinary bladder dysfunction, which leads to pure urge urinary incontinence or mixed urinary incontinence, exacerbating co-existing stress urinary incontinence. Previous studies suggest that an onset of detrusor overactivity after radical prostatectomy seems to be a significant biological risk factor, which is related to urinary incontinence after surgery. ${ }^{7}$ An overactive bladder negatively impacts quality of life and often requires pharmacotherapy. A study by Blaivas et al. revealed that about $20 \%$ of men who developed OAB symptoms were previously treated for prostate cancer. ${ }^{8}$ Another study by Porena et al. observed detrusor overactivity in patients after radical prostatectomy in about $2-77 \%$ of cases during urodynamic testing. ${ }^{9}$ Boettcher et al. found that patients after radical prostatectomy and radiotherapy had OAB 3 years after the therapy in approx. $13 \%$ and $30 \%$ of cases, respectively. ${ }^{10}$ The data suggests that patients with prostate cancer after radical prostatectomy are at a higher risk of developing OAB. Hosier et al. revealed that $\mathrm{OAB}$ and storage LUTS are quite common in patients after radical prostatectomy. ${ }^{11}$ Additionally, adjuvant or salvage radiotherapy increases the risk of OAB in patients primarily treated with radical prostatectomy. In about $4 \%$ of cases, detrusor overactivity leads to urge urinary incontinence in patients after radical prostatectomy. In about $42 \%$ of cases, detrusor overactivity is also associated with mixed urinary incontinence after radical prostatectomy. ${ }^{12}$ The results from Song et al. showed that 
more than $51 \%$ of patients presented with detrusor overactivity at a 3-year follow-up after radical prostatectomy. ${ }^{13}$ It is well-known that radical prostatectomy may affect proper innervation and vascularization of the urinary bladder, thus causing a functional alteration of the urinary bladder, such as detrusor overactivity and/or decreased urinary bladder compliancy.

Considering the number of factors that potentially affect urinary continence after radical prostatectomy, it can be inferred that post-prostatectomy urinary continence is an unpredictable and clinically demanding condition. Thus, the proper evaluation of these potential factors in patients after radical prostatectomy is crucial in predicting the risk and severity of urinary incontinence development. A better understanding of the etiopathogenesis of urinary incontinence in relation to radical prostatectomy will enable physicians to more thoroughly inform patients about the risks associated with urinary incontinence, along with potential treatment options for the symptoms they are experiencing.

\section{Bladder outlet obstruction and overactive bladder}

Benign prostate enlargement is a common urological problem mainly seen in older men, causing LUTS, such as nocturia, frequency, urgency, etc. It is known that BOO, depending on the severity and duration of the obstruction, leads to structural changes in the urinary bladder. A significant proportion of patients with benign prostate enlargement require treatment due to the discomfort caused by LUTS. In patients with benign prostate enlargement, about $60 \%$ of symptomatic patients and $52 \%$ of asymptomatic patients experience urinary BOO. Although urodynamics is an invasive, expensive and time-consuming procedure, it still remains the most valuable test for making a final diagnosis of urinary BOO. Therefore, new methods of evaluating urinary $\mathrm{BOO}$ are required. So far, the following clinical factors were defined as predictors for urinary BOO: 1. International Prostate Symptom Score (IPSS) >19 points; 2. maximal urine flow $\left(\mathrm{Q}_{\max }\right)<15 \mathrm{~mL} / \mathrm{min}$; and 3. post-void residual volume $>100 \mathrm{~mL}$. In addition, a study by Güzel et al. suggested that the urinary bladder wall thickness (BWT) increases simultaneously with increasing urinary BOO. ${ }^{14}$ The authors of this study showed that the measurement of BWT is a simple, fast and reproducible procedure predicting the severity of urinary BOO. Hakenberg et al. revealed that men with LUTS and benign prostate enlargement presented with a moderate increase in BWT. ${ }^{15}$

Based on clinical experience, many patients with prostate cancer have co-existing benign prostate enlargement with or without BOO. Thus, this relationship may predispose a patient to develop $\mathrm{OAB}$ and urinary incontinence, especially following radical prostatectomy. Therefore, it is crucial to identify obstructed patients who seem to be at a higher risk of developing post-prostatectomy storage
LUTS, especially urge urinary incontinence, which is due to OAB development.

\section{Urinary bladder ultrasonographic features may predict overactive bladder}

Ultrasonography is crucial in diagnosing functional urinary bladder disorders. The BWT parameter is useful in OAB diagnosis. Previous studies have revealed that an increased BWT is a predictor of detrusor overactivity in patients diagnosed with OAB ${ }^{16}$ An increased BWT seems to be a consequence of increased intensity of detrusor muscle contractions against a competent urethral sphincter, ultimately leading to urinary bladder hypertrophy. Yang and Huang reported that a thickened urinary bladder wall is common in women with lower urinary tract symptoms. ${ }^{17}$ A study by Khullar et al. found the average BWT to be $5 \mathrm{~mm} .{ }^{18}$ This mean value was important in the creation of a sensitive screening method for diagnosing detrusor overactivity in symptomatic women without urinary bladder outflow obstruction. A study by Ali et al. suggested that an evaluation of BWT in ultrasonography is a very sensitive and specific technique for a diagnosis of detrusor overactivity. ${ }^{19}$ The sensitivity and specificity of this test amount to about $91.9 \%$ and $86.1 \%$, respectively.

\section{Study design}

To test the hypothesis, we have decided to conduct an experiment to investigate the effect of urinary bladder structural changes and urinary $\mathrm{BOO}$ on $\mathrm{OAB}$ and urinary incontinence development in patients after radical prostatectomy. The study is designed for patients with prostate cancer who qualified for radical prostatectomy. Currently, the study is still ongoing. Before surgery, each patient is strictly evaluated for $\mathrm{OAB}$ and $\mathrm{BOO}$. After that, all patients are assigned to one of the following study groups: group 1 - patients with no OAB symptoms and without BOO; group 2 - patients with no OAB symptoms and with $\mathrm{BOO}$; group 3 - patients with $\mathrm{OAB}$ symptoms and with $\mathrm{BOO}$; and group $4-$ patients with OAB symptoms and without BOO. Additionally, as a reference experimental group (control group), we enrolled 30 healthy, young volunteers with no OAB symptoms and without benign prostate enlargement or BOO. All patients with prostate cancer who qualified for radical prostatectomy are evaluated. Before surgery, the patients fill in the IPSS questionnaire, which assesses LUTS. Overactive bladder symptoms are analyzed using King's questionnaire, as well as 2 different OAB symptom scores (OABSS) designed by Blaivas et al. and Homma et al. ${ }^{20-22}$ Each patient undergoes a transabdominal ultrasonographic evaluation of the prostate and urinary bladder structure. The width (W), height $(\mathrm{H})$, length $(\mathrm{L})$, and volume of the prostates are recorded. In each patients the ultrasonography is done when normal desire to void is achieved. The BWT and detrusor 
muscle thickness (DWT) are measured in the sagittal plane in the anterior bladder wall. The means of 3 measurements of BWT and DWT are calculated. The urinary bladder weight - Ultrasound Estimated Bladder Weight (UEBW) - is also estimated. Ultrasound Estimated Bladder Weight is calculated from the urinary bladder surface area (SA), BWT and specific gravity of the urinary bladder wall $(\delta)$, using the following formula: $\mathrm{UEBW}=\mathrm{SA} \times \mathrm{BWT} \times \delta .{ }^{22-24}$ The urinary bladder wall has a specific gravity of $1 \mathrm{~g} / \mathrm{cm}^{3}$. After ultrasonography, each patient undergoes uroflowmetry with post-void residual estimation. The same procedures are carried out in healthy subjects, as control, for the exclusion of benign prostate enlargement, BOO and OAB symptoms. Based on the protocol described above, all patients are divided into aforementioned 4 study groups. The study is going to be continued until each experimental group includes at least 30 patients. The study has been approved by the Local Ethical Committee of Ludwik Rydygier Collegium Medicum in Bydgoszcz, Nicolaus Copernicus University in Toruń, Poland..

The inclusion criteria for the patients in the study are as follows: 1 . adult men over 40 years of age with prostate cancer who qualified for radical prostatectomy; 2 . the control group comprised of 30 healthy men aged $20-40$ years without any urological conditions and with no LUTS. The exclusion criteria are as follows: 1. neoadjuvant (any time before) or adjuvant radiotherapy or brachytherapy (within the follow-up period of 12 months); 2. macroscopic infiltration of the urinary bladder (pT4); 3. neurological deficiencies (e.g., stroke, spinal cord injury, multiple sclerosis, Parkinson's disease, etc.) which would cause an inability to control the urinary bladder; 4 . urethral strictures; 5 . previous surgery, such a transurethral resection of the prostate (TURP) or adenomectomy, performed within 12 months prior to radical prostatectomy; 6 . any form of constant catheterization (an indwelling catheter, clean intermittent catheterization); 7. any bowel dysfunction (especially constipation, irritable bowel syndrome (IBS), etc.); and 8. taking a medication affecting urine production (e.g., diuretics).

After the initial evaluation prior to surgery, the control evaluation is set for $1,3,6,9$, and 12 months postoperatively in groups 1-4 in order to define LUTS after radical prostatectomy in relation to pre-surgical patient clinical features.

Our study was entitled "Effects of urinary bladder hypertrophy on urge urine incontinence after radical prostatectomy" and was registered at the website https://clinicaltrials.gov (ID number: NCT03061760).

\section{Implications}

The possible impact of urinary bladder hypertrophy on $\mathrm{OAB}$ with or without urge urinary incontinence in patients after radical prostatectomy may point at potential new methods for the prevention and control of OAB and urinary incontinence after surgery. Some possible methods are oral pharmacotherapy or intravesical onabotulinumtoxin A injections in pre-prostatectomy mode in the cases of patients with urinary bladder hypertrophy.

\section{Consequences of the hypothesis}

The data and evidence presented in this paper support the idea that fluctuations in BWT can predict the development of OAB. Thus, we hypothesized that urinary bladder hypertrophy influences the development of urine incontinence in patients with prostate cancer after radical prostatectomy through the occurrence of OAB. This provides a better method of classification of patients with a higher risk of developing urge urinary incontinence after radical prostatectomy.

In conclusion, it would be beneficial to conduct further studies to confirm whether urinary bladder hypertrophy is a sensitive predictor of $\mathrm{OAB}$ and/or urge urinary incontinence in the general population, as well as in patients after radical prostatectomy.

\section{References}

1. Arnold M, Karim-Kos HE, Coebergh JW, et al. Recent trends in incidence of five common cancers in 26 European countries since 1988: Analysis of the European Cancer Observatory. Eur J Cancer. 2015;51(9):1164-1187.

2. Bianco FJ, Scardino PT, Eastham JA. Radical prostatectomy: Longterm cancer control and recovery of sexual and urinary function ("trifecta"). Urology. 2005;66(5):83-94.

3. Kelleher CJ, Resse PR, Pleil AM, Okano GJ. Health-related quality of life of patients receiving extended-release tolterodine for overactive bladder. Am J Manag Care. 2002;8(Suppl 19):S608-S615.

4. AdamczykP, Tworkiewicz J, Drewa T. Radical prostatectomy specimens: A voice against focal therapy. Cent European JUrol. 2014;67(3):235-241.

5. Ficarra V, Novara G, Rosen RC, et al. Systematic review and meta-analysis of studies reporting urinary continence recovery after roboticassisted radical prostatectomy. Eur Urol. 2012;62(3):405-417.

6. Pastore AL, Palleschi G, Illiano E, Zucchi A, Carbone A, Costantini E. The role of detrusor overactivity in urinary incontinence after radical prostatectomy: A systemic review. Minerva Urol Nefrol. 2017;69(3): 234-241.

7. Heesakkers J, Farag F, Bauer RM, Sandhu J, De Ridder D, Stenzl A. Pathophysiology and contributing factors in postprostatectomy incontinence: A review. Eur Urol. 2017;71(6):936-944.

8. Blaivas JG, Marks BK, Weiss JP, Panagopoulos G, Somaroo C. Differential diagnosis of overactive bladder in men. J Urol. 2009;182(6): 2814-2817.

9. Porena M, Mearini E, Mearini L, Vianello A, Giannantoni A. Voiding dysfunction after radical retropubic prostatectomy: More than external urethral sphincter deficiency. Eur Urol. 2007;52(1):38-45.

10. Boettcher M, Haselhuhn A, Jakse G, Brehmer B, Kirschner-Hermanns R. Overactive bladder syndrome: An underestimated longterm problem after treatment of patients with localized prostate cancer? BJU Int. 2012;109(12):1824-1830.

11. Hosier GW, Tennankore KK, Himmelman JG, Gajewski J, Cox AR. Overactive bladder and storage lower urinary tract symptoms following radical prostatectomy. Urology. 2016;94:193-7. doi: 10.1016/j.urology.2016.05.007

12. Novara G, Ficarra V, D'Elia C, et al. Evaluating urinary continence and preoperative predictors of urinary continence after robot-assisted laparoscopic radical prostatectomy. J Urol. 2010;184(3):1028-1033. 
13. Song C, Lee J, Hong JH, Choo MS, Kim CS, Ahn H. Urodynamic interpretation of changing bladder function and voiding pattern after radical prostatectomy: A long-term follow-up. BJU Int. 2010;106(5): 681-686.

14. Güzel Ö, Aslan Y, Balci M, et al. Can bladder wall thickness measurement be used for detecting bladder outlet obstruction? Urology. 2015;86(3):439-444.

15. Hakenberg OW, Linne C, Manseck A, Wirth MP. Bladder wall thickness in normal adults and men with mild lower urinary tract symptoms and benign prostatic enlargement. Neurourol Urodyn. 2000;19(5): 585-593.

16. Kuo HC. Measurement of detrusor wall thickness in women with overactive bladder by transvaginal and transabdominal sonography. Int Urogynecol J Pelvic Floor Dysfunct. 2009;20(11):1293-1299.

17. Yang JM, Huang WC. Bladder wall thickness on ultrasonographic cystourethrography: Affecting factors and their implications. J Ultrasound Med. 2003;22(8):777-782.

18. Khullar V, Cardozo LD, Salvatore S, Hill S. Ultrasound: A noninvasive screening test for detrusor instability. Br JObstet Gynaecol. 1996;103(9): 904-908.
19. Ali MM, Ahmed A-F, Khaled SM, Abozeid H, AbdelMagid ME. Accuracy of ultrasound-measured bladder wall thickness for diagnosis of detrusor overactivity. African J Urol. 2015;21(1):25-29.

20. Lin YT, Chou ECL. Assessment of overactive bladder (OAB): Symptom scores. Incont Pelvic Floor Dysfunct. 2009;3(Suppl 1):9-14.

21. Blaivas JG, Panagopoulos G, Weiss JP, Somaroo C. Validation of the overactive bladder symptom score. J Urol. 2007;178(2):543-547.

22. Homma Y, Yoshida M, Seki N, et al. Symptom assessment tool for overactive bladder syndrome: Overactive bladder symptom score. Urology. 2006;68(2):318-323.

23. Kojima M, Inui E, Ochiai A, Naya Y, Ukimura O, Watanabe H. Ultrasonic estimation of bladder weight as a measure of bladder hypertrophy in men with infravesical obstruction: A preliminary report. Urology. 1996;47(6):942-947.

24. Chalana V, Dudycha S, Yuk JT, McMorrow G. Automatic measurement of ultrasound-estimated bladder weight (UEBW) from three-dimensional ultrasound. Rev Urol. 2005;7(Suppl 6):S22-S28. 